Research Paper

\title{
Gustave Roussy Immune Score (GRIm-Score) is a prognostic marker in patients with resectable esophageal squamous cell carcinoma
}

\author{
Ji-Feng Feng ${ }^{1,2}{ }^{\bowtie}$, Liang Wang ${ }^{1}$, Xun Yang ${ }^{1,2}$, Sheng Chen ${ }^{1 凶}$ \\ 1. Department of Thoracic Oncological Surgery, Institute of Cancer Research and Basic Medical Sciences of Chinese Academy of Sciences, Cancer Hospital of \\ University of Chinese Academy of Sciences, Zhejiang Cancer Hospital, No.38 Guangji Road, Hangzhou 310022, China. \\ 2. Key Laboratory Diagnosis and Treatment Technology on Thoracic Oncology, No.38 Guangji Road, Zhejiang province, Hangzhou 310022, China. \\ $\square$ Corresponding authors: Ji-Feng Feng and Sheng Chen. Department of Thoracic Oncological Surgery, Institute of Cancer Research and Basic Medical Sciences \\ of Chinese Academy of Sciences, Cancer Hospital of University of Chinese Academy of Sciences, Zhejiang Cancer Hospital, No.38 Guangji Road, Hangzhou
} 310022, China. E-mail: Fengj@ @jcc.org.cn (Feng); Chengsheng@zjcc.org.cn (Chen). Tel: +86-0571-88128072.

(c) The author(s). This is an open access article distributed under the terms of the Creative Commons Attribution License (https://creativecommons.org/licenses/by/4.0/). See http://ivyspring.com/terms for full terms and conditions.

Received: 2019.06.25; Accepted: 2019.11.20; Published: 2020.01.01

\begin{abstract}
Background: The Gustave Roussy Immune Score (GRIm-Score) was initially reported to select patients for immunotherapy. Therefore, the purpose of the current retrospective study was to determine whether GRIm-Score, a novel nutritional and inflammatory-based prognostic score, is a useful prognostic marker in patients with esophageal squamous cell carcinoma (ESCC) undergoing surgical resection.

Methods: A retrospective single institutional study including 372 ESCC patients undergoing surgical resection was performed. The GRIm-Score was simply calculated by lactate dehydrogenase (LDH), neutrophil lymphocyte ratio (NLR) and albumin (ALB). The cancer-specific survival (CSS) was analyzed for the current study with Cox regression analyses with forward stepwise and Kaplan-Meier methods.

Results: There were 284 (76.3\%) men and 88 (23.7\%) women with the mean age of $59.3 \pm 8.0$ years (range: $36-80$ years). Patient with a high GRIm-Score had poor CSS ( $10.3 \%$ vs. $35.0 \%, P<0.001)$. The GRIm-Score, in multivariate analyses, instead of NLR, LDH or ALB, was an independent prognostic factor for CSS $(P=0.004)$.

Conclusion: The GRIm-Score was an independent prognostic marker in patients with ESCC undergoing surgical resection. Our study is also the first study to discuss the prognostic value of GRIm-Score in patients with resectable ESCC.
\end{abstract}

Key words: esophageal squamous cell carcinoma, neutrophil to lymphocyte ratio, albumin, lactate dehydrogenase, prognosis

\section{Introduction}

As one of the leading malignant tumors worldwide, esophageal cancer (EC) is common in China with 477,900 new cases and 375,000 deaths [1]. Esophageal squamous cell carcinoma (ESCC) is the main pathological type of EC in China [2]. Although there are many therapies for patients with ESCC, the prognosis for patients with ESCC remains poor [3,4]. Surgical resection remains the first choice for patients with early-stage disease, while chemoradiotherapy is the mainstay treatment in patients with local advanced disease. Therefore, it is very necessary to find more useful and effective preoperative clinical variables for patients with ESCC.

The nutrition and inflammation play an important role in cancer prognosis [5]. Some studies revealed that neutrophil to lymphocyte ratio (NLR) and C-reactive protein (CRP) were associated with prognosis in several types of cancers, including EC 
[6-9]. As an important nutritional factor, albumin (ALB) reflected the nutritional status in a variety of cancers. Some studies published in recent years revealed that ALB was still a controversial prognostic factor in patients with EC [10,11]. It has been reported that lactate dehydrogenase (LDH) may act as an indicator for tumor burden and aggressiveness [12, 13]. Patients with high level of serum LDH have worse prognosis, but remains controversial in ESCC [14-16].

Recently, the Gustave Roussy Immune Score (GRIm-Score) was firstly identified with the purpose of a better patient selection in clinical trials for immunotherapy [17]. The results demonstrated that the GRIm-Score, based on NLR, LDH and ALB, is a better prognostic marker for patients enrolled in experimental trials. Moreover, the prognostic value of GRIm-Score has been confirmed in non-small cell lung cancer [18, 19]. However, as far as we know, there are no studies regarding the prognostic value of GRIm-Score in patients with ESCC so far. The purpose of the current study was to evaluate the prognostic value of GRIm-Score in patients with resectable ESCC.

\section{Materials and Methods}

\section{Patients}

We retrospectively analyzed the clinical characteristics of 372 resectable patients with ESCC who undergoing curative esophagectomy between January 2006 and December 2010. This was a single institutional study. The inclusion criteria were as follow: (1) ESCC with stage I-III was confirmed by histopathology, (2) curative esophagectomy was performed without any neoadjuvant chemoradiotherapy, and (3) clinical characteristics and preoperative laboratory results, such as neutrophil, lymphocyte, LDH, ALB and CRP, were obtained before surgery within one week. The study was approved by the Ethics Committees, and written informed consent for the collection of specimen was obtained from each patient. The last follow up time was June 2014.

\section{Data collection}

The main clinical characteristics requiring analysis were collected from our medical records. The levels of neutrophil, lymphocyte, LDH, ALB and CRP were obtained within one week before surgery. The 7th AJCC/UICC TNM staging system was used in the current study [20]. The GRIm-Score is calculated by the following three variables: LDH (within normal range: 0 vs. $>$ upper limit of normal (ULN) of each center, $240 \mathrm{U} / \mathrm{L}$ in our hospital: +1$)$, ALB ( $\geq 35 \mathrm{~g} / \mathrm{L}: 0$ vs. $<35$ g/L: +1$)$, and NLR ( $\leq 6: 0$ vs. $>6:+1)$. Patients were divided into two groups: high group (score 2 or 3 ) and low group (score 0 or 1) [17].

\section{Statistical analyses}

The Chi-squared test (for categorical variables), Student's t-test (for continuous variables with Gaussian distribution) and Mann-Whitney U test (for continuous variables without Gaussian distribution) were performed to compare the clinical characteristics grouped by GRIm-Score. The endpoint for the current study was cancer-specific survival (CSS). The Kaplan-Meier method was used to compare the CSS by using the log-rank test. Multivariate analyses with Cox regression analyses by forward stepwise regression were used to evaluate the independent prognostic factors. The areas under the curve (AUC) for NLR, LDH, ALB, CRP and GRIm-Score were compared and calculated with the receiver operating characteristic (ROC) curves. A nomogram model with $\mathrm{R}$ 3.6.0 software was used to predict the 1-, 3- and 5 -year CSS probability by using the independent prognostic factors in multivariate analyses [21]. All statistical analyses were performed with MedCalc 15.2 (MedCalc Software bvba, Ostend, Belgium) and SPSS 20.0 (SPSS Inc., Chicago, IL, USA).

\section{Results}

\section{Patient Characteristics}

There were $284(76.3 \%)$ men and 88 (23.7\%) women with the mean age of $59.3 \pm 8.0$ years (range: 36-80 years). According to clinical criteria, a total of 115 patients $(30.9 \%)$ received postoperative adjuvant radiotherapy and/or chemotherapy. The histograms of the NLR, LDH and ALB were shown in Figure 1. There were negative correlations between NLR and ALB $(\mathrm{r}=-0.169, P=0.001$; Fig. 2A), positive correlations between NLR and LDH $(r=0.174, P=$ 0.001; Fig. 2B), but no correlations between ALB and LDH $(r=-0.078, P=0.135$; Fig. 2C). The clinical characteristics regarding GRIm-Score were shown in Table 1.

\section{CSS analyses}

At the last follow-up time, 256 (68.8\%) of the 372 patients had died. Patients with a high GRIm-Score had poor 5-year CSS (10.3\% vs. 35.0\%, $P<0.001)$ (Fig. 3A). In subgroup analyses based on TNM stage (TNM I, TNM II and TNM III), we revealed that GRIm-Score was also significantly related to CSS in TNM II $(P=$ $0.020)$ and TNM III $(P=0.011)$, but not in TNM I $(P=$ 0.334) (Fig. 3B-D). The significantly differences for 5 -year CSS were also found in NLR $(34.4 \%$ vs. $10.2 \%$, $P<0.001)$, ALB $(35.0 \%$ vs. $15.3 \%, P<0.001), \mathrm{LDH}$ 
( $35.8 \%$ vs. $16.7 \%, P<0.001)$ and CRP $(36.7 \%$ vs. $15.5 \%$, $P<0.001$ ) (Fig. 3E-H).

Table 1. Clinical characteristics based on GRIm-Score in patients with ESCC.

\begin{tabular}{|c|c|c|c|c|}
\hline & Total $(\mathrm{n}=372)$ & Low $(n=314)$ & $\operatorname{High}(n=58)$ & P-value \\
\hline \multicolumn{5}{|l|}{ Age (years) } \\
\hline Mean \pm SD & $59.3 \pm 8.0$ & $59.5 \pm 8.0$ & $58.3 \pm 7.7$ & $0.322^{\#}$ \\
\hline$\leq 60 />60(\mathrm{n})$ & $210 / 162$ & $176 / 138$ & $34 / 24$ & 0.717 \\
\hline \multicolumn{5}{|l|}{ Gender (n) } \\
\hline \multicolumn{5}{|l|}{ Tumor length $(\mathrm{cm})$} \\
\hline Median (IQR) & $4.0(3.0-5.3)$ & $4.0(3.0-5.0)$ & $5.0(4.0-6.3)$ & $<0.001^{*}$ \\
\hline \multicolumn{4}{|l|}{ Tumor location (n) } & 0.017 \\
\hline \multicolumn{4}{|l|}{ Vessel invasion (n) } & 0.632 \\
\hline \multicolumn{4}{|l|}{ Perineural invasion (n) } & 0.802 \\
\hline $\begin{array}{l}\text { Negative / Positive } \\
\text { Smoking (n) }\end{array}$ & \multicolumn{3}{|c|}{ Smoking (n) } & 0.348 \\
\hline \multicolumn{5}{|l|}{ Drinking (n) } \\
\hline \multicolumn{4}{|l|}{ Differentiation (n) } & 0.872 \\
\hline $\begin{array}{l}\text { Well/ Moderate/ Poor } \\
\text { TNM stage (n) }\end{array}$ & \multicolumn{3}{|c|}{ TNM stage (n) } & 0.266 \\
\hline $\begin{array}{l}\text { I / II / III } \\
\text { NLR }\end{array}$ & 94 / 119 / 159 & $91 / 102 / 121$ & $3 / 17 / 38$ & $<0.001$ \\
\hline Median (IQR) & $2.93(2.16-3.87)$ & $2.74(1.95-3.67)$ & $6.32(4.09-7.09)$ & $<0.001^{*}$ \\
\hline \multicolumn{4}{|l|}{ LDH (U/L) } & $<0.001$ \\
\hline Mean \pm SD & $187.0 \pm 66.1$ & $177.2 \pm 62.7$ & $240.1 \pm 58.7$ & $<0.001^{\#}$ \\
\hline $\begin{array}{l}\leq 240 />240(\mathrm{n}) \\
\operatorname{ALB}(\mathrm{g} / \mathrm{L})\end{array}$ & \multicolumn{3}{|c|}{$\operatorname{ALB}(\mathrm{g} / \mathrm{L})$} & $<0.001$ \\
\hline Mean \pm SD & $40.9 \pm 5.5$ & $41.7 \pm 5.1$ & $36.7 \pm 5.6$ & $<0.001^{\#}$ \\
\hline$>35 / \leq 35(\mathrm{n})$ & $300 / 72$ & $284 / 30$ & $16 / 42$ & $<0.001$ \\
\hline \multicolumn{5}{|l|}{ CRP (mg/L) } \\
\hline Median (IQR) & $4.55(1.96-10.26)$ & $4.461 .72-8.39)$ & $7.52(3.43-15.58)$ & $<0.001^{*}$ \\
\hline $\begin{array}{l}\leq 10 />10(\mathrm{n}) \\
\text { Adjuvant therapy }\end{array}$ & 275 / 97 & $242 / 72$ & $33 / 25$ & $<0.001$ \\
\hline No / Yes & $257 / 115$ & $220 / 94$ & $37 / 21$ & 0.342 \\
\hline
\end{tabular}

ESCC: esophageal squamous cell carcinoma; GRIm-Score: Gustave Roussy Immune Score; CRP: C-reactive protein; ALB: albumin; LDH: lactate dehydrogenase; NLR: neutrophil to lymphocyte ratio; TNM: tumor node metastasis; SD: standard

deviation; IQR: interquartile range. "\#: t-test; *: Mann-Whitney U test.

\section{Cox regression analyses}

Univariate analyses indicated that several clinical indexes, such as vessel invasion, perineural invasion, tumor length, TNM stage, CRP, ALB, NLR, LDH and GRIm-Score, were significant predictors of CSS (Table 2). Multivariate analyses demonstrated that GRIm-Score (HR: 1.593, 95\% CI: 1.156-2.197, P = 0.004), instead of NLR, LDH or ALB, was an independent prognostic factor (Table 3). Furthermore, CRP (HR: 1.760, 95\% CI: 1.339-2.314, $P<0.001$ ) and TNM stage (HR: 1.478, 95\% CI: 1.027-2.129, $P=0.036$ and HR: 2.364, 95\% CI: 1.676-3.332, $P<0.001)$ were other significant prognostic factors (Table 3 ).

\section{ROC curve analyses}

The AUC areas were 0.644, 0.596, 0.564, 0.572 and 0.582 for GRIm-Score (95\% CI: 0.593-0.693), CRP (95\% CI: 0.544-0.646), NLR (95\% CI: 0.512-0.615), ALB
(95\% CI: 0.520-0.623) and LDH (95\% CI: 0.530-0.632), respectively (Fig. 4). Comparison of AUC areas regarding the GRIm-Score, CRP, NLR, ALB and LDH in ESCC were shown in Table 4.

Table 2. Univariate analyses of CSS in ESCC patients.

\begin{tabular}{|c|c|c|c|c|c|}
\hline & 5-year CSS & Mediean (M) & P-value & HR $(95 \%$ CI) & $P$-value \\
\hline Age (years) & & & 0.997 & & 0.997 \\
\hline$\leq 60$ & $30.5 \%$ & 32 & & 1.000 & \\
\hline$>60$ & $32.1 \%$ & 32 & & $1.000(0.780-1.280)$ & \\
\hline Gender & & & 0.950 & & 0.951 \\
\hline female & $31.8 \%$ & 28 & & 1.000 & \\
\hline male & $31.0 \%$ & 32 & & $1.009(0.756-1.348)$ & \\
\hline Tumor length (cm) & & & 0.003 & & 0.004 \\
\hline$\leq 3.0$ & $39.6 \%$ & 48 & & 1.000 & \\
\hline$>3.0$ & $27.8 \%$ & 27 & & $1.522(1.147-2.021)$ & \\
\hline Tumor location & & & 0.381 & & 0.392 \\
\hline upper & $44.0 \%$ & 45 & & 1.000 & \\
\hline middle & $29.1 \%$ & 32 & & $1.471(0.846-2.558)$ & 0.172 \\
\hline lower & $31.4 \%$ & 32 & & $1.402(0.806-2.439)$ & 0.231 \\
\hline Vessel invasion & & & 0.015 & & 0.017 \\
\hline negative & $33.3 \%$ & 36 & & 1.000 & \\
\hline positive & $20.0 \%$ & 20 & & $1.469(1.073-2.013)$ & \\
\hline Perineural invasion & & & 0.006 & & 0.007 \\
\hline negative & $34.1 \%$ & 39 & & 1.000 & \\
\hline positive & $20.3 \%$ & 20 & & $1.486(1.117-1.977)$ & \\
\hline Smoking & & & 0.265 & & 0.271 \\
\hline no & $34.0 \%$ & 35 & & 1.000 & \\
\hline yes & $27.8 \%$ & 27 & & $1.148(0.898-1.467)$ & \\
\hline Drinking & & & 0.606 & & 0.610 \\
\hline no & $30.3 \%$ & 36 & & 1.000 & \\
\hline yes & $32.6 \%$ & 25 & & $1.068(0.830-1.375)$ & \\
\hline Differentiation & & & 0.142 & & 0.150 \\
\hline well & $38.5 \%$ & 35 & & 1.000 & \\
\hline moderate & $30.9 \%$ & 34 & & $1.118(0.766-1.631)$ & 0.564 \\
\hline poor & $27.0 \%$ & 16 & & $1.461(0.943-2.263)$ & 0.089 \\
\hline TNM stage & & & $<0.001$ & & $<0.001$ \\
\hline I & $48.9 \%$ & 67 & & 1.000 & \\
\hline II & $33.6 \%$ & 34 & & $1.697(1.185-2.430)$ & 0.004 \\
\hline III & $18.9 \%$ & 19 & & $2.784(1.995-3.886)$ & $<0.001$ \\
\hline CRP (mg/L) & & & $<0.001$ & & $<0.001$ \\
\hline$\leq 10.0$ & $36.7 \%$ & 42 & & 1.000 & \\
\hline$>10.0$ & $15.5 \%$ & 17 & & $2.060(1.581-2.685)$ & \\
\hline $\operatorname{ALB}(g / L)$ & & & $<0.001$ & & $<0.001$ \\
\hline$\geq 35.0$ & $35.0 \%$ & 40 & & 1.000 & \\
\hline$<35.0$ & $15.3 \%$ & 20 & & $1.979(1.480-2.645)$ & \\
\hline NLR & & & $<0.001$ & & $<0.001$ \\
\hline$\leq 6.0$ & $34.4 \%$ & 36 & & 1.000 & \\
\hline$>6.0$ & $10.2 \%$ & 20 & & $2.029(1.464-2.812)$ & \\
\hline $\mathrm{LDH}(\mathrm{U} / \mathrm{L})$ & & & $<0.001$ & & $<0.001$ \\
\hline$\leq 240$ & $35.8 \%$ & 39 & & 1.000 & \\
\hline$>240$ & $16.7 \%$ & 20 & & $1.767(1.348-2.315)$ & \\
\hline GRIm-Score & & & $<0.001$ & & $<0.001$ \\
\hline low & $35.0 \%$ & 39 & & 1.000 & \\
\hline high & $10.3 \%$ & 18 & & $2.246(1.652-3.054)$ & \\
\hline Adjuvant therapy & & & 0.129 & & 0.133 \\
\hline No & $32.3 \%$ & 38 & & 1.000 & \\
\hline Yes & $28.7 \%$ & 23 & & $1.223(0.940-1.591)$ & \\
\hline
\end{tabular}

ESCC: esophageal squamous cell carcinoma; CSS: cancer-specific survival; GRIm-Score: Gustave Roussy Immune Score; CRP: C-reactive protein; ALB albumin; LDH: lactate dehydrogenase; TNM: tumor node metastasis; NLR: neutrophil to lymphocyte ratio; CI: confidence interval; HR: hazard ratio.

\section{ROC curve analyses}

The AUC areas were $0.644,0.596,0.564,0.572$ and 0.582 for GRIm-Score (95\% CI: 0.593-0.693), CRP (95\% CI: 0.544-0.646), NLR (95\% CI: 0.512-0.615), ALB (95\% CI: 0.520-0.623) and LDH (95\% CI: 0.530-0.632), 
respectively (Fig. 4). Comparison of AUC areas regarding the GRIm-Score, CRP, NLR, ALB and LDH in ESCC were shown in Table 4.

Table 3. Multivariate analyses of CSS in patients with ESCC.

\begin{tabular}{lll}
\hline & HR $(95 \% \mathrm{CI})$ & P-value \\
\hline TNM stage & $<0.001$ \\
I & 1.000 & \\
II & $1.478(1.027-2.129)$ & 0.036 \\
III & $2.364(1.676-3.332)$ & $<0.001$ \\
GRIm-Score & & 0.004 \\
low & 1.000 & \\
high & $1.593(1.156-2.197)$ & \\
CRP $(\mathrm{mg} / \mathrm{L})$ & & \\
$\leq 10.0$ & 1.000 & \\
$>10.0$ & $1.760(1.339-2.314)$ & \\
\end{tabular}

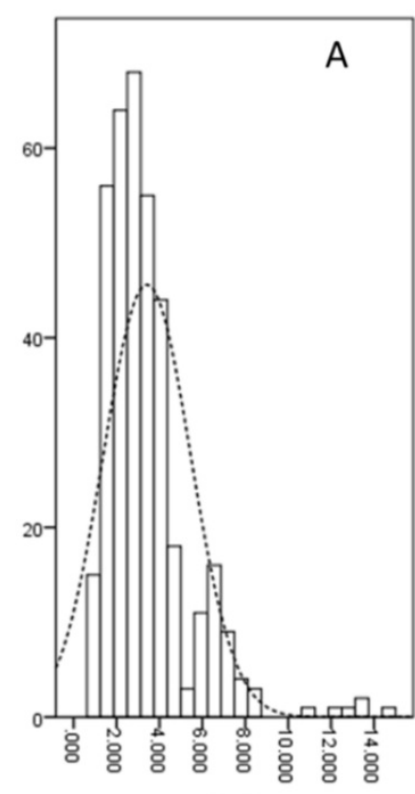

NLR

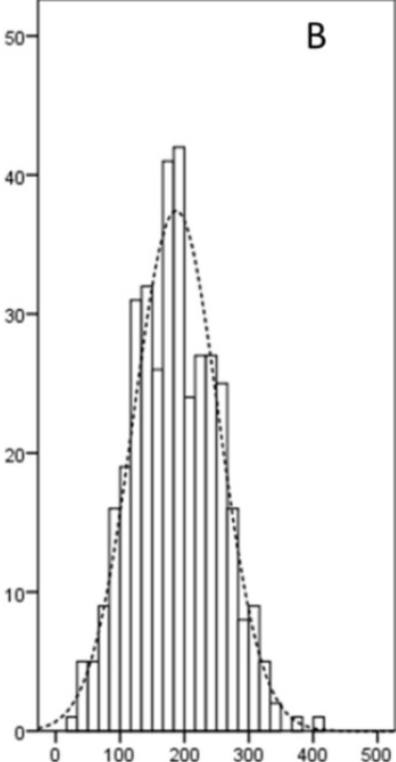

LDH

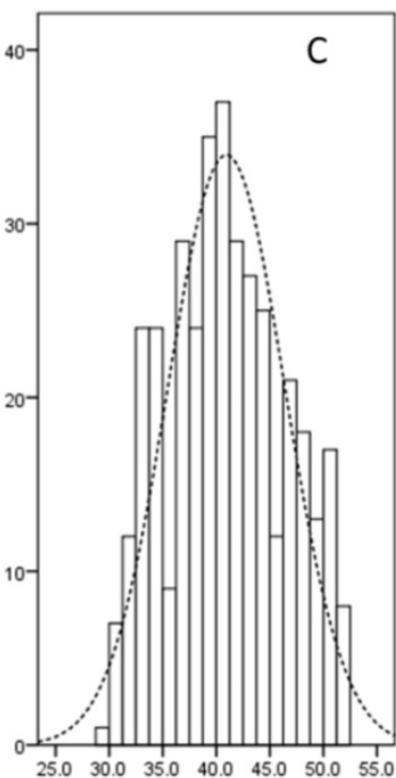

ALB

Figure 1. The histograms of the NLR (A), LDH (B) and ALB (C)
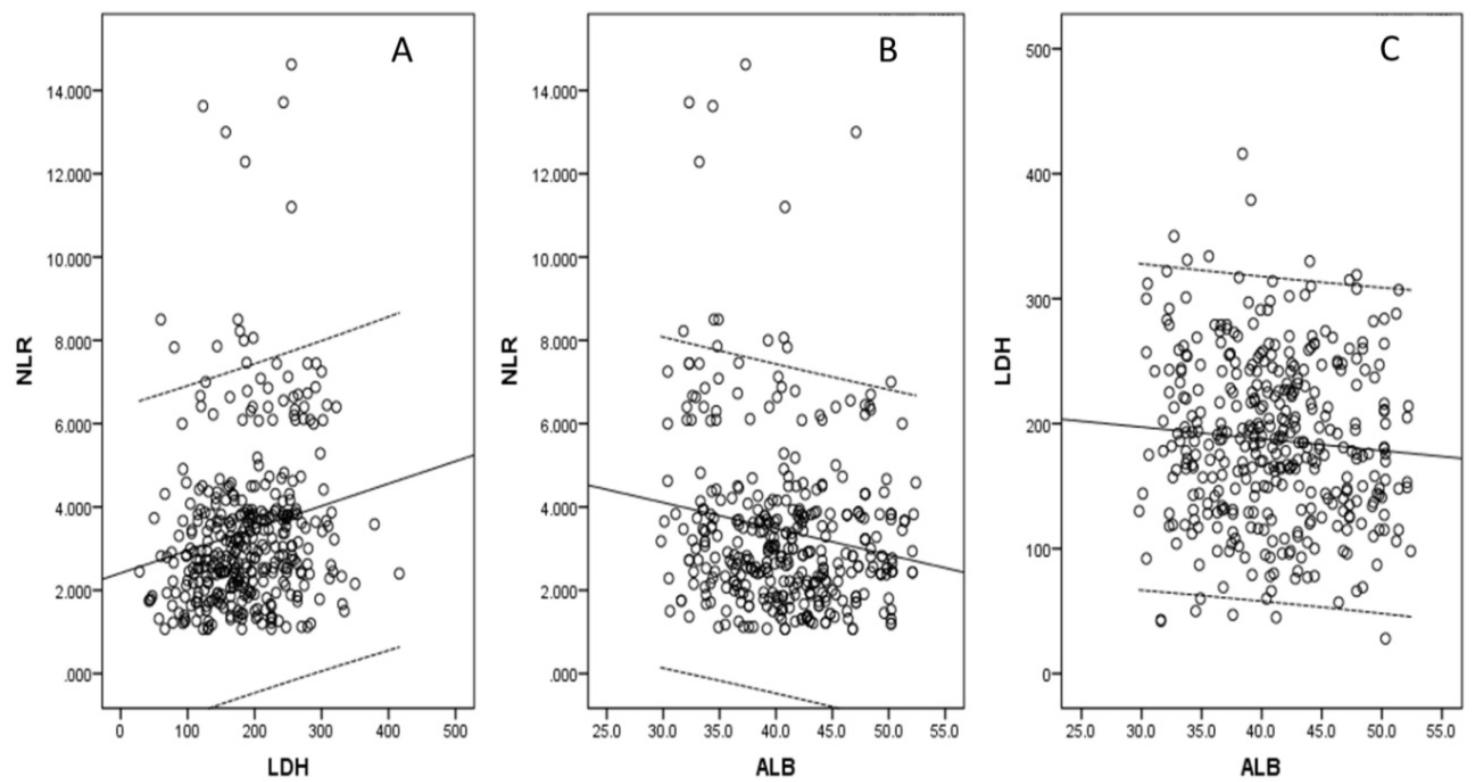

Figure 2. Correlations for NLR, LDH and ALB. Negative correlations between NLR and ALB (A). Positive correlations between NLR and LDH (B). No correlations were found between ALB and LDH (C). 

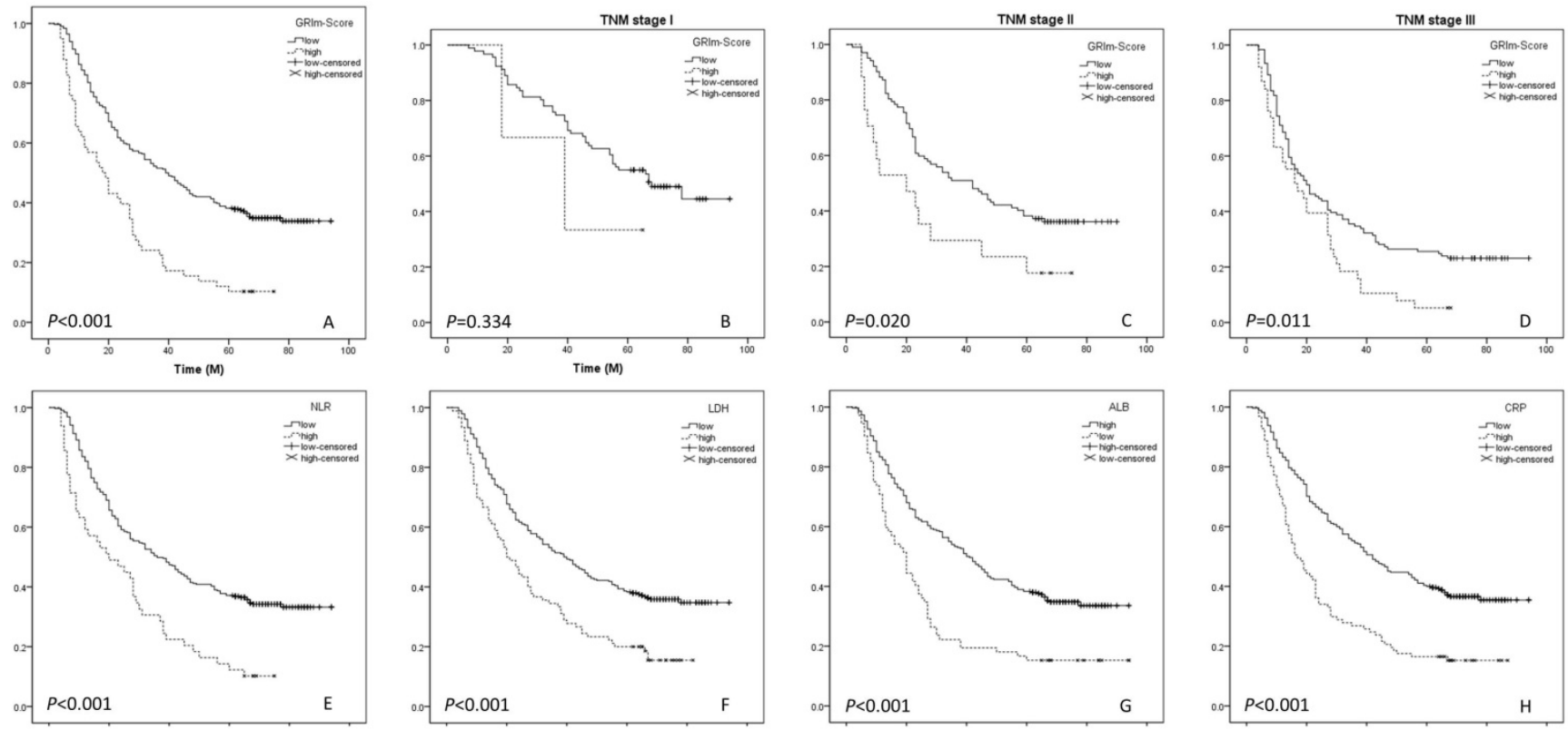

Figure 3. CSS analyses. Kaplan-Meier for CSS grouped by GRIm-Score (A). CSS analyses for GRIm-Score in subgroup analyses based on TNM stage (B-D). Kaplan-Meier for CSS grouped by NLR (E), LDH (F), ALB (G) and CRP $(H)$.

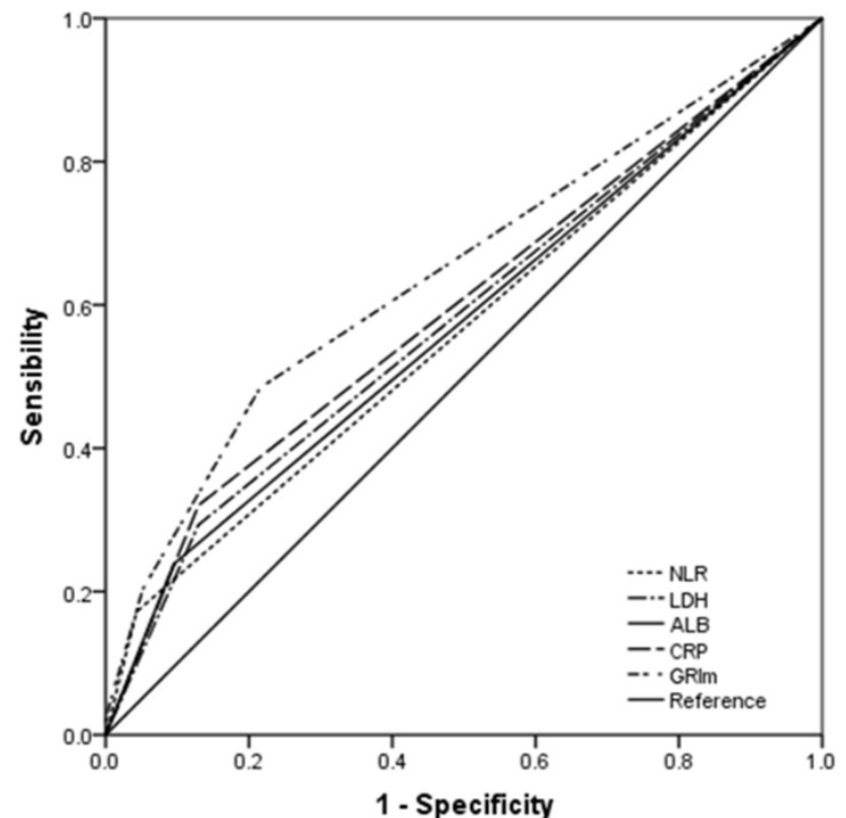

Figure 4. ROC analyses. The AUC area of the GRIm-Score (0.644) was higher than that of CRP (0.596), NLR (0.564), ALB (0.572) and LDH (0.582) for all the ESCC patients.

\section{Nomogram analyses}

Moreover, a nomogram model, including the significant prognostic factors (GRIm-Score, TNM and CRP), was conducted to predict the 1-, 3- and 5-year CSS probability for patients with ESCC (Fig. 5). Two nomogram models were conducted for different GRIm-Score (GRIm-Score 0, 1 and GRIm-Score 0, 1, 2 and 3 ).

\section{Discussion}

This study, as far as we know, is the first study in patients with ESCC to indicate the prognostic value of GRIm-Score. The current study revealed several important findings: (1) GRIm-Score was a strong prognostic marker for CSS; (2) GRIm-Score, instead of NLR, ALB or LDH, was a useful independent prognostic factor. Therefore, we concluded that the GRIm-score was not only a selective biomarkers for patients enrolled in clinical trials for immunotherapy, but also a useful prognostic biomarkers for ESCC patients undergoing surgical resection.

The GRIm-Score was firstly identified with the purpose of a better patient selection in clinical trials for immunotherapy [17]. The results demonstrated that the GRIm-Score, based on NLR, LDH and ALB, is a better prognostic marker for patients enrolled in experimental trials. Moreover, the prognostic value of GRIm-Score has been confirmed in non-small cell lung cancer in recent years $[18,19]$. However, as far as we know, there are no studies regarding the prognostic value of GRIm-Score in ESCC patients so far. In our study, patients with a high GRIm-Score had worse 5 -year CSS $(10.3 \%$ vs. $35.0 \%, P<0.001)$. Multivariate analyses demonstrated that GRIm-Score (HR: 1.593, 95\% CI: 1.156-2.197, $P=0.004$ ), instead of NLR, LDH or ALB, was an independent prognostic factor.

The NLR, ALB and LDH are routine laboratory indicators in daily clinical practice. Recent studies reported that inflammation is associated with poor prognosis in cancers, with NLR as a sensitive 
inflammatory biomarker in several types of cancers, including EC [6-9]. The levels of ALB, as an important nutritional factor, reflected the nutritional status in a variety of cancers. Some studies published in recent years revealed that ALB was still a controversial prognostic factor in patients with EC [10,11]. Patients with high level of serum LDH have worse prognosis, but remains controversial in ESCC [14-16]. A retrospective study including 906 patients with ESCC concluded that $\mathrm{LDH}$ was associated with overall survival (OS) with the optimal cutoff point of 361.5 U/L [14]. However, a total of 212 patients with ESCC undergoing chemoradiotherapy revealed that $\mathrm{LDH}$ (cutoff point: $170 \mathrm{U} / \mathrm{L}$ ) was not associated with OS or progression-free survival (PFS) [15]. The results were consistent with another study including 447 patients with ESCC which revealed that LDH (cutoff point: $154.4 \mathrm{U} / \mathrm{L}$ ) was not a prognostic factor regarding OS [16].
It should be noted that NLR, ALB and LDH, routine laboratory indicators in daily clinical practice, may be influenced by various conditions. In the present study, although NLR, ALB and LDH were found to be predictive factors for CSS in univariate analyses, multivariate analyses did not show these biomarkers to be independent prognostic factors. Therefore, GRIm-Score is a combined indicator which can reflect a mixed prognostic value.

It should be also noted that our results have potential clinical significance in the treatment of patients with ESCC. Patients in a high level of GRIm-Score with early-stage ESCC may require more frequent follow-up, while those with local advanced ESCC may need more adjuvant therapy. However, the results of our study should be validated in more large-sample prospective trials in future.

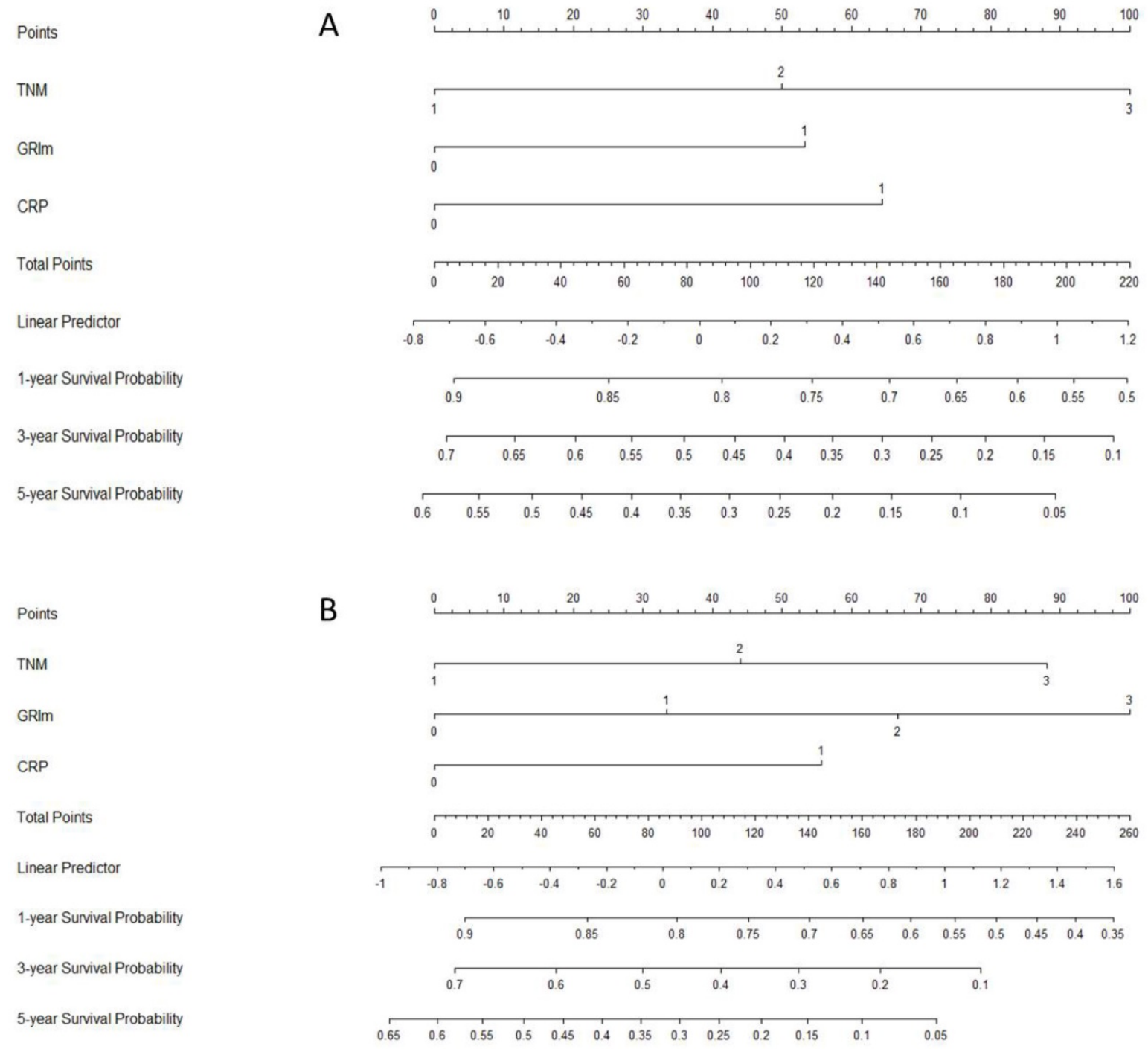

Figure 5. Nomogram analyses. Two nomogram models were conducted for different GRIm-Score [GRIm-Score 0, 1 (A) and GRIm-Score 0, 1, 2, 3 (B)] to predict the 1-, 3and 5-year CSS probability. 
Some potential limitations of the current study should be mentioned. Firstly, the potential selection bias should be acknowledged due to the current study was a retrospective research with single center. Secondly, patients with preoperative treatment in the current study were excluded, which might have influenced the results for patients with ESCC who underwent surgical resection. As everyone knows, preoperative neoadjuvant therapy will have side effects on laboratory indicators. However, preoperative neoadjuvant therapy (chemotherapy and/or radiotherapy) followed by surgery improves survival in several randomized control trials for locally advanced disease of EC, but not for early-stage EC $[22,23]$. Thirdly, due to lack of prospective study, the results of our study should be validated in more large-sample clinical trials in future. In the current study, a nomogram model, including the significant prognostic factors (GRIm-Score, TNM and CRP), was conducted to predict the 1-, 3- and 5-year CSS probability for patients with ESCC. However, we must acknowledge that our prognostic nomogram model was only developed, but not validated in this study. Therefore, the results of our study should be validated in future.

In summary, the GRIm-Score, a novel nutritional and inflammatory-based prognostic score, was an independent prognostic factor in patients with ESCC undergoing surgical resection. The current study, as far as we know, is the first study regarding the prognostic value of GRIm-Score in ESCC patients undergoing esophagectomy.

\section{Acknowledgments}

This study was funded by the Medical Health Science and Technology Project of Zhejiang Provincial Health Commission (NO. 2018KY290, 2019RC129 and 2019RC128).

\section{Competing Interests}

The authors have declared that no competing interest exists.

\section{References}

1. Chen W, Zheng R, Baade PD, Zhang S, Zeng H, Bray F, et al. Cancer statistics in China, 2015. CA: Cancer J Clin. 2016; 66: 115-32.

2. Lin Y, Totsuka Y, He Y, Kikuchi S, Qiao Y, Ueda J, et al. Epidemiology of esophageal cancer in Japan and China. J Epidemiol. 2013; 23: 233-42.

3. Napier KJ, Scheerer M, Misra S. Esophageal cancer: A review of epidemiology, pathog enesis, staging workup and treatment modalities. World J Gastrointest Oncol. 2014; 6: 112-20.

4. Bedenne L, Michel P, Bouché O, Milan C, Mariette C, Conroy T, et al. Chemoradiation followed by surgery compared with chemoradiation alone in squamous cancer of the esophagus: FFCD 9102. J Clin Oncol. 2007; 25: 1160-8.

5. Mantovani A, Allavena P, Sica A, Balkwill F. Cancer-related inflammation. Nature. 2018; 24: 436-44.

6. Shimura T, Kitagawa M, Yamada T, Ebi M, Mizoshita T, Tanida S, et al. $\mathrm{C}$-reactive protein is a potential prognostic factor for metastatic gastric cancer. Anticancer Res. 2012; 32: 491-6.
7. Zingg U, Forberger J, Rajcic B, Langton C, Jamieson GG. Association of C-reactive protein levels and long-term survival after neoadjuvant therapy and esophagectomy for esophageal cancer. J Gastrointest Surg. 2010; 14: 462-9.

8. Chen MF, Chen PT, Kuan FC, Chen WC. The predictive value of pretreatment neutrophil-to-lymphocyte ratio in esophageal squamous cell carcinoma. Ann Surg Oncol. 2019; 26: 190-9.

9. Sato Y, Gonda K, Harada M, Tanisaka Y, Arai S, Mashimo Y, et al. Increased neutrophil-to-lymphocyte ratio is a novel marker for nutrition, inflammation and chemotherapy outcome in patients with locally advanced and metastatic esophageal squamous cell carcinoma. Biomed Rep. 2017; 7: 79-84.

10. Miyata H, Yamasaki M, Kurokawa Y, Takiguchi S, Nakajima K, Fujiwara Y, et al. Prognostic value of an inflammation-based score in patients undergoing preoperative chemotherapy followed by surgery for esophageal cancer. Exp Ther Med. 2011; 2: 879-85.

11. Kobayashi T, Oshima K, Yokobori T, Idetsu A, Hayashi Y, Hinohara RN, et al. Perioperative nutriture in esophageal cancer patients undergoing esophagectomy. Hepatogastroenterology. 2013; 60: 1311-6.

12. Gong T, Liu J, Jiang J, Zhai YF, Wu CM, Ma C, et al. The role of lactate deshydrogenase levels on non-small cell lung cancer prognosis: a meta-analysis. Cell Mol Biol. 2019; 65: 89-93.

13. Yu SL, Xu LT, Oi O, Geng YW, Chen $H$, Meng ZQ, et al. Serum lactate dehydrogenase predicts prognosis and correlates with systemic inflammatory response in patients with advanced pancreatic cancer after gemcitabine-based chemotherapy. Sci Rep. 2017; 7: 45194.

14. Wei $X L$, Zhang DS, He MM, Jin Y, Wang DS, Zhou YX, et al. The predictive value of alkaline phosphatase and lactate dehydrogenase for overall survival in patients with esophageal squamous cell carcinoma. Tumour Biol. 2016; 37: 1879-87.

15. Zhang P, Xi M, Li QQ, He LR, Liu SL, Zhao L, et al. The modified Glasgow prognostic score is an independent prognostic factor in patients with inoperable thoracic esophageal squamous cell carcinoma undergoing chemoradiotherapy. J Cancer. 2014; 5: 689-95

16. Huang $\mathrm{H}$, Wang XP, Li XH, Chen H, Zheng X, Lin JH, et al. Prognostic value of pretreatment alanine aminotransferase/aspartate aminotransferase (ALT/AST) ratio and gamma glutamyltransferase (GGT) in patients with esophageal squamous cell carcinoma. BMC Cancer. 2017; 17: 544.

17. Bigot F, Castanon E, Baldini C, Hollebecque A, Carmona A, Postel-Vinay S, et al. Prospective validation of a prognostic score for patients in immunotherapy phase I trials: The Gustave Roussy Immune Score (GRIm-Score). Eur J Cancer. 2017; 84: 212-8.

18. Minami S, Ihara S, Ikuta S, Komuta K. Gustave Roussy Immune Score and Royal Marsden Hospital Prognostic Score are biomarkers of Immune-Checkpoint Inhibitor for non-small cell lung cancer. World J Oncol. 2019;10: 90-100.

19. Minami S, Ihara S, Komuta K. Gustave Roussy Immune Score is a prognostic factor for chemotherapy-naive pulmonary adenocarcinoma with wild-type epidermal growth factor receptor. World J Oncol. 2019; 10: 55-61.

20. Rice TW, Rusch VW, Ishwaran $\mathrm{H}$, Blackstone $\mathrm{EH}$, Worldwide Esophageal Cancer Collaboration. Cancer of the esophagus and esophagogastric junction: data-driven staging for the seventh edition of the American Joint Committee on Cancer/International Union Against Cancer Staging Manuals. Cancer. 2010; 116: 3763-73.

21. Iasonos A, Schrag D, Raj GV, Panageas KS. How to build and interpret a nomogram for cancer prognosis. J Clin Oncol. 2008; 26: 1364-1370.

22. Shapiro J, van Lanschot JJB, Hulshof MCCM, van Hagen $P$, van Berge Henegouwen MI, Wijnhoven BPL, et al. Neoadjuvant chemoradiotherapy plus surgery versus surgery alone for oesophageal or junctional cancer (CROSS): long-term results of a randomised controlled trial. Lancet Oncol. 2015; 16: $1090-8$

23. Mariette C, Dahan L, Mornex F, Maillard E, Thomas PA, Meunier B, et al. Surgery alone versus chemoradiotherapy followed by surgery for stage I and II esophageal cancer: final analysis of randomized controlled phase III trial FFCD 9901. J Clin Oncol. 2014; 32: 2416-22. 